\title{
Guillain-Barre syndrome occurring in the course of dengue fever
}

Sir,

Infections with various organisms have been reported preceding Guillain-Barre syndrome (GBS). Recent infections with Campylobacter jejuni, cytomegalovirus, Epstein-Barr virus, and Mycoplasma pneumoniae are specifically related to GBS. ${ }^{[1]}$ We report a case of GBS occurring in the course of dengue fever (DF).

A 40-year-old man presented with fever, myalgias, severe backache, and skin rash of 3-day duration. There was no history suggestive of mucocutaneous bleeding. Clinical examination was essentially normal. Investigations showed hemoglobin of $11.2 \mathrm{~g} \%$, total leukocytes count $4100 / \mathrm{mm}^{3}$ and platelet count $30000 / \mathrm{mm}^{3}$. Specific IgM antibody against dengue virus was present. PCR for dengue was also positive. He was conservatively managed. There were no acute complications of dengue virus infection. Seven days later, the patient developed acute-onset weakness of all four limbs associated with areflexia. Power was Grade 3/5 in all four limbs. There was no clinical involvement of cranial nerves, sensory system, or autonomic nervous system. Electrophysiological studies showed grossly reduced amplitudes of all motor and sensory nerves, distal latency, and conduction velocity being normal. $F$-wave latencies were prolonged. Needle EMG showed fibrillation potentials and positive sharp waves suggestive of acute denervation. Cerebrospinal fluid analysis showed 5 leukocytes $/ \mathrm{mm}^{3}$, protein $130 \mathrm{mg} \%$, and sugar $98 \mathrm{mg} \%$. A diagnosis of GBS - acute motor-sensory axonal neuropathy (AMSAN) variant - was made and a course of intravenous immunoglobulins was given. The patient made a significant recovery and was able to walk 3 weeks later.

Guillain-Barre syndrome has been reported following a variety of bacterial and viral infections. These infections are not uniquely associated with any clinical subtype but severe axonal degeneration is more common following $C$. jejuni and severe sensory impairment following cytomegalovirus. ${ }^{[1]}$ Dengue fever as an antecedent infection in GBS is uncommon. ${ }^{[2],[3]}$ To the best of our knowledge, this association has not been reported from India.

Guillain-Barre syndrome has a wide clinical spectrum, ranging from mild self-limiting disease to acute fulminant cases with severe pandysautonomia. Electrophysiologically too, axonal and demyelinating varieties are seen. These variations are probably dependent on both the antecedent infection and host immune status. Currently, efforts to correlate the clinical picture with the inciting infection have not succeeded. The previous cases of GBS associated with dengue virus were clinically severe, requiring mechanical ventilation and were demy- 
elinating in nature; ${ }^{[2],[3]}$ however, our patient had milder disease and the GBS was of axonal type. Thus, dengue virus infection too does not seem to result in any specific pattern of GBS. However, all the reported cases survived and recovered with appropriate therapy.

In conclusion, in patients presenting with GBS in whom no usual antecedent infections are identified, screening for dengue virus infection may help in identifying a rare cause.

\section{Sudhir Kumar, Subhashini Prabhakar}

Division of Neurology, Department of Neurological Sciences, Apollo Hospitals, Jubilee Hills, Hyderabad, India. E-mail: drsudhirkumar@yahoo.com

\section{References}

1. Hughes RA, Hadden RD, Gregson NA, Smith K.J. Pathogenesis of GuillainBarre syndrome. J Neuroimmunol 1999;100:74-97.

2. Santos NQ, Azoubel AC, Lopes AA, Costa G, Bacellar A. Guillain-Barre syndrome in the course of dengue: Case report. Arq Neuropsiquiatr 2004;62:1446.

3. Esack A, Teelucksingh S, Singh N. The Guillain-Barre syndrome following dengue fever. West Indian Med .J 1999;48:36-7.

Accepted on 13-11-2004 